\title{
Phase-contrast microscopy at high X-ray energy with a laboratory set-up
}

\author{
Marco Endrizzi, ${ }^{1, *}$ Fabio A. Vittoria, ${ }^{1,2}$ Paul C. Diemoz, ${ }^{1,2}$ Rodolfo Lorenzo, ${ }^{3}$ Robert \\ D. Speller, ${ }^{1}$ Ulrich Wagner, ${ }^{4}$ Christoph Rau, ${ }^{4}$ lan K. Robinson, ${ }^{5,2}$ and Alessandro Olivo ${ }^{1,2}$ \\ ${ }^{1}$ Department of Medical Physics and Bioengineering, University College London, \\ Gower Street, WC1E 6BT London, United Kingdom \\ ${ }^{2}$ Research Complex at Harwell, Harwell Oxford Campus, OX11 OFA Didcot, United Kingdom \\ ${ }^{3}$ Department of Civil Engineering, University College London, \\ Gower Street, WC1E 6BT London, United Kingdom \\ ${ }^{4}$ Diamond Light Source, Harwell Oxford Campus, OX11 ODE Didcot, United Kingdom \\ ${ }^{5}$ London Centre for Nanotechnology, WC1H OAH London, United Kingdom
}

compiled: April 10, 2014

\begin{abstract}
We report on the design and realization of an X-ray imaging system for quantitative phase-contrast microscopy at high X-ray energy, with laboratory-scale instrumentation. Phase and amplitude were separated quantitatively at X-ray energies up to $80 \mathrm{keV}$ with micrometric spatial resolution. The accuracy of the results was tested against numerical simulations and the spatial resolution was experimentally quantified by measuring a Siemens star phase object. This simple set-up should find broad application in those areas of X-ray imaging where high energy and spatial resolution are simultaneously required and in those difficult cases where the sample contains materials with similar X-ray absorption.
\end{abstract}

Hard X-ray radiation has a high penetration power in matter, thus enabling non-destructive inspection of the inner structure of samples. At the same time, this power is also a weakness because the contrast arising from differences in the absorption of radiation tends to vanish when partially transparent samples are illuminated. X-ray phase-contrast imaging (XPCi) [1] overcomes this problem because it is sensitive to the phase shifts imparted to the X-ray wave when traversing the sample. Several methods $[2-15]$ have been developed for performing XPCi. Approaches have also been proposed for high-resolution XPCi, including freespace propagation, Zernike phase contrast and gratingbased methods [16-22]. Here we show the design, modelling and realization of a laboratory system based on the edge-illumination $[10,15]$ principle and implemented through its area-imaging counterpart, sometimes referred to as the coded-aperture [23] method. These are non-interferometric methods that do not use Talbot self imaging effect or Moire patterns [24]; contrast is generated by fine angular selection, in analogy with analyser based imaging which uses the rocking curve of a crystal [25]. We aim to push the current resolution limits of a few tens of micrometers [26] towards microscopic resolution, while still performing quantitative phase-contrast imaging at high X-ray energies. A magnified projection

\footnotetext{
* Corresponding author: m.endrizzi@ucl.ac.uk
}

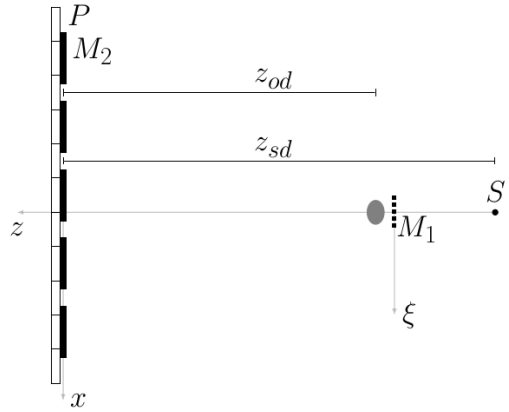

Fig. 1. Experimental set-up: the X-ray beam, generated by a microfocus X-ray source $S$, is shaped by the first mask $M_{1}$, traverses the sample and is analysed by the second mask $M_{2}$ before being recorded by the pixels $P$ of a digital detector.

geometry was used in order to achieve high spatial resolution while being able to efficiently detect the radiation. The ability of the method to be quantitative and its spatial resolution are experimentally demonstrated and numerically simulated while the potential of the technique in terms of image quality is illustrated through images of a complex wood sample.

The experimental set-up consists of a microfocus transmission target X-ray tube, two apertured masks and a detector (Fig. 1). The tungsten target X-ray tube is operated at $80 \mathrm{kVp}$ and has a focus of $3.5 \mu \mathrm{m}$. The first mask $M_{1}$ is placed at $13 \mathrm{~cm}$ from the focus and the 
source to detector distance is $z_{s d}=130 \mathrm{~cm}$. The sample is positioned at about $14 \mathrm{~cm}$ from the focus, with a geometrical magnification factor $G=z_{s d} /\left(z_{s d}-z_{o d}\right)$ of about 9 . The first mask $M_{1}$ has a pitch $p_{1}=20 \mu \mathrm{m}$ and apertures $a_{1}=3 \mu \mathrm{m}$ while $p_{2}=98 \mu \mathrm{m}$ and $a_{2}=29 \mu \mathrm{m}$ are used for the second mask $M_{2}$. They are made of gold on a graphite (M2) and silicon (M1) substrate and were manufactured by Creatv Microtech (Potomac, MD) and Microworks GmbH (Karlsruhe, Germany), respectively. The detector is a passive pixel CMOS flat panel sensor (Hamamatsu Photonics C9732DK), with pixel pitch $p_{3}=50 \mu \mathrm{m}$. The signal degradation due to cross-talk between neighbouring pixels is limited by the use of a line-skipped mask design [27]. The main limiting factor for the field of view is $a_{1}$, which defines the angular acceptance of the transmitted radiation $\theta_{m} \approx a_{1} / t_{M_{1}}$, where $t_{M_{1}}$ is the thickness of the mask, about $200 \mu \mathrm{m}$ in this case. For our set-up the field of view was $2 \times 5$ $\mathrm{mm}$, in the $x$ and $y$ directions, respectively. Bent masks could be used for obtaining a larger field of view with an even more compact set-up [28]. Provided that the angular spread of the beam is limited to $\theta_{l}<8 p_{3} / z_{\text {od }} \sim 340$ $\mu \mathrm{rad}$, the pixels can be considered independent from one another, which allows us to model the image formation for a single aperture only.

Let us consider the Fourier transform of the intensity $I_{D}(x)=\int d f \exp (2 \pi i x f) \hat{I}_{D}(f)$, on the detector [29]

$$
\hat{I}_{D}(f)=\int d x^{\prime} e^{-2 \pi i x^{\prime} f} T\left(x^{\prime}-w\right) T^{*}\left(x^{\prime}+w\right)
$$

where $w=z_{o d} \lambda f /(2 G)$ and $T(x)=$ $A(x) \exp [-M(x)] \exp [-i \phi(x)]$ combines the aperture transmission function $A(x)$ and the sample absorption and phase shift. If not differently specified, the integration is always carried out between $-\infty$ and $\infty$. The signal detected is given by $S=\int_{0}^{a_{2}} d x I_{D}(x) \approx \int_{0}^{\infty} d x I_{D}(x)$ assuming $a_{2}$ wider that the beam extension. Using $\int_{0}^{\infty} d x \exp (2 \pi i x f)=(1 / 2) \delta(f)-[1 /(2 \pi i f)]$, where $\delta(f)$ represents the Dirac delta function, $S$ can be expressed as a sum of two terms $S=S_{1}+S_{2} . S_{1}=\int d x^{\prime}\left|T\left(x^{\prime}\right)\right|^{2}$ and

$$
S_{2}=-\int d x^{\prime} \int d f \frac{e^{-2 \pi i x^{\prime} f}}{2 \pi i f} T\left(x^{\prime}-w\right) T^{*}\left(x^{\prime}+w\right) .
$$

The integration of $S_{2}$ can be split into two parts $\int_{-\infty}^{\infty} d f=\int_{-\infty}^{0} d f+\int_{0}^{\infty} d f$ and rearranged into a single integral according to the properties of symmetry possessed by the integrand. The first term $S_{1}$ depends only on absorption while the second one $S_{2}$ is also related to phase. This can be shown explicitly by assuming constant absorption within the limits defined by the sample aperture and considering $\left|\phi\left(x^{\prime}+w\right)-\phi\left(x^{\prime}-w\right)\right|<<1$ [30]. The following form for $S_{2}$ is obtained:

$$
S_{2}=\frac{e^{-2 M}}{\pi} \int_{0}^{\frac{a_{1}}{2}} d w \int_{-b_{1}}^{b_{1}} d x^{\prime} \frac{\Delta \phi_{x^{\prime} w}}{w} \cos \left[4 \pi \frac{x^{\prime} w G}{\lambda z_{o d}}\right]
$$

where $\Delta \phi_{x^{\prime} w}=\phi\left(x^{\prime}-w\right)-\phi\left(x^{\prime}+w\right), b_{1}=\left(a_{1}-2 w\right) / 2$ and the limits of integrations have been redefined according to the transmission function of the sample aperture. This expression provides a means to study how the phase signal is modulated by the imaging system. The component of the signal related to the phase effects is given by the weighted sum of all the possible difference quotients within the limits defined by the aperture. The weak phase condition used to derive Eq. 3 is required only to obtain a formulation easier to interpret, without the need to carry out the full integration numerically. In order to visualize this, the $S_{2}$ signal was calculated for a sinusoidal phase object, and its maximum value plotted against the period in Fig. 2. It is interesting to note that

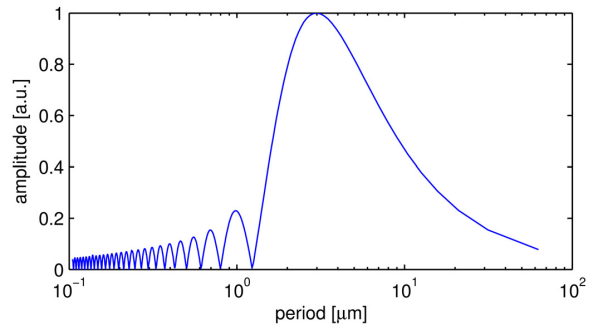

Fig. 2. Evaluation of Eq. 3 as a function of the period of a sinusoidal phase object.

the modulation is peaked at $3 \mu \mathrm{m}$, which corresponds to $a_{1}$, and also that signals with period smaller than the aperture are detected; for example, $S_{2}$ is reduced by about $50 \%$ at $1.5 \mu \mathrm{m}$.

The direct comparison between the measured and the theoretical signal from a star pattern test object is carried out by means of a numerical simulation of the whole imaging system [31]. Each monochromatic component was weighted according to the X-ray source spectrum and the detector response as function of energy [32]. In order to perform quantitative retrieval of the absorption and refraction of the sample, we followed the approach based on the knowledge of the translation curve (TC) of the system [25]. The TC describes how the detected intensity changes as a function of the displacement $\Delta \xi$ between the two masks. Images were acquired by setting the displacement between $M_{1}$ and $M_{2}$ as equal to $\Delta \xi_{1}=-\Delta \xi_{2}=2 \mu \mathrm{m}$. The resulting images can be expressed as [25]:

$$
I_{L, R}=\exp \left[-\int_{\mathcal{O}} \bar{\mu} d z\right] I_{T C}\left(\Delta \xi_{1,2}-z_{o d} \bar{\phi}_{x} / G\right)
$$

where $\bar{\mu}$ denotes the linear attenuation coefficient of the sample evaluated at the effective energy of the system [33], $z_{o d}$ is the sample-to-detector distance, $\bar{\phi}_{x}=\partial \bar{\phi} / \partial x$ is the partial derivative of $\phi$ at the effective energy of the system, and $I_{T C}$ is the value of the translation curve. We also introduce the following images $I_{\Sigma}=I_{L}+I_{R}$ and $I_{\Delta}=I_{L}-I_{R}[25]$. It is worth noting that the quantity $I_{\Delta} / I_{\Sigma}$ depends only upon $\bar{\phi}_{x}$ and the operational parameters of the set-up [25]: we will refer to this quantity 


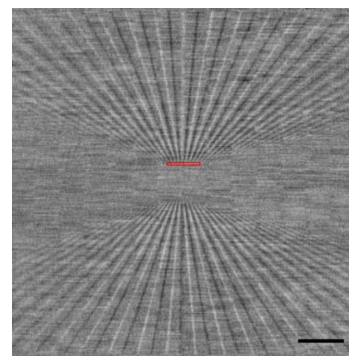

(a)

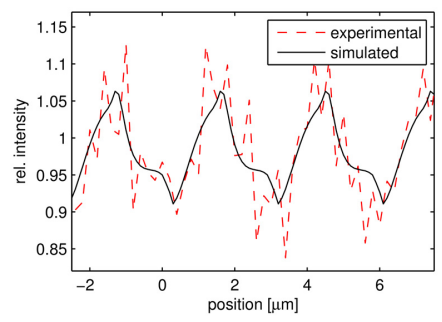

(b)
Fig. 3. Star pattern phase object. (a) differential phasecontrast experimental image (scale bar is $50 \mu \mathrm{m}$ ). (b) the simulation (solid line) compared against the experimental profile (dashed line) extracted from the highlighted region in the centre of the star.

as the differential phase image. Moreover, $I_{\Sigma}$ depends only on the absorption properties of the sample. The following samples were imaged experimentally: a star pattern, a cylindrical two-material sample and a bamboo wood sample. The star pattern was etched into a few hundred microns of crystalline silicon and provides a good approximation of a pure phase object, at the X-ray energies we used. The $I_{L}$ image was acquired by using 100 sample scan steps of $0.2 \mu \mathrm{m}$, with an exposure time of $70 \mathrm{~s}$ per step. The cylindrical sample was composed of two materials, $220 \mu \mathrm{m}$ diameter of boron with a $14 \mu \mathrm{m}$ diameter tungsten core, and it was scanned with 16 steps of $1.2 \mu \mathrm{m}, 100 \mathrm{~s}$ exposure time each. The bamboo wood was sliced to a thickness of about $500 \mu \mathrm{m}, I_{L}$ and $I_{R}$ were acquired scanning the sample with 44 steps of $0.5 \mu \mathrm{m}$, with an exposure time of $100 \mathrm{~s}$ per step. For comparison, an image of the bamboo sample was also acquired in free-space propagation XPCi, with monochromatic synchrotron radiation and a high-resolution detector. The image was acquired at the I13 beamline of the Diamond Light Source by using $9.7 \mathrm{keV}$ X-rays and a detector featuring $0.8 \mu \mathrm{m}$ size pixels. The sample was placed at about $200 \mathrm{~m}$ from the undulator source and the detector $30 \mathrm{~cm}$ downstream of the sample.

The spatial resolution of the laboratory-based system is evaluated by means of the star pattern. The image of the star is shown in Fig. 3(a) from which the intensity profile shown in Fig. 3(b) was extracted (dashed line). The experimental data are in good agreement with the numerical simulation, shown as a solid line in the plot in Fig. 3(b). As the absorption is negligible in this case, we note from Eq. 4 that $I_{L}$ depends only on $\bar{\phi}_{x}$ and on the operational parameters of the set-up. The changing visibility of the phase-contrast features around the star pattern are a property of the geometry of the setup. Let us consider the phase shift of the star pattern as a replica at various angles $\varphi$ of a step-like feature; the detected signal is modulated by a cosine function, $S_{2} \propto \cos (\varphi) \partial_{u} \phi(u, v)$, where $(u, v)$ are the $(x, y)$ coordinates rotated by the angle $\varphi$. The smallest resolved separation between a dark and a bright fringe is $1.5 \mathrm{mi}-$

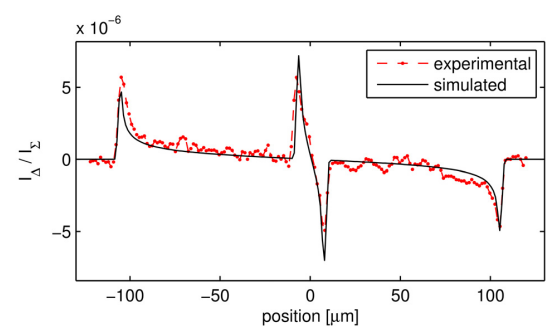

(a)

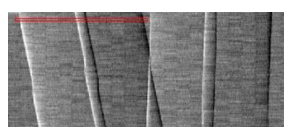

(b)

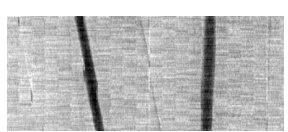

(c)
Fig. 4. Boron fibre with a tungsten core sample. (a) comparison of the experimental intensity profile (highlighted region in (b) sector) against the theoretically expected one for a single fibre. (b) differential phase and (c) amplitude images of two fibres at an angle.

crons; the sample used hardly allowed testing finer resolutions. The quantitativeness of the method is demonstrated by retrieving differential phase and amplitude of the boron fiber with a tungsten core and comparing the extracted (dashed line) values to the expected ones (solid line) in Fig. 4(a). The theoretical $\bar{\phi}_{x}(x)$ was calculated assuming a cylindrical shape of the boron and tungsten fiber. The analytical profile was convolved with a Gaussian function, representing the spatial resolution of the system, of width $1.5 \mu \mathrm{m}$. The refractive indices of the materials were calculated by using the Xraylib library [34]. The profile was then obtained according to $I_{\Delta} / I_{\Sigma}=\left(z_{o d} \bar{\phi}_{x} / G\right) /\left(I_{T C}^{\prime}(\Delta \xi) / I_{T C}(\Delta \xi)\right)[25]$ by numerically differentiating $\bar{\phi}$. The amplitude and differential phase images are also shown in Fig. 4(c) and 4(b), respectively. The horizontal line noise that can be noticed in Fig. 3(a), 4(b) and 4(c) is a result of the brick-like structure used to manufacture M1. The laboratory images of the bamboo sample are shown in Fig. 5, where the contributions from phase (Fig. 5(a)) and absorption (Fig. 5(b)) are separated. For a better appreciation of the image quality, the image acquired under nearly ideal

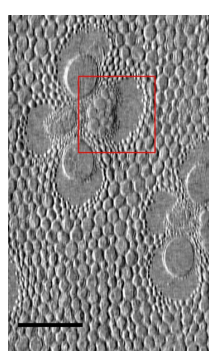

(a)

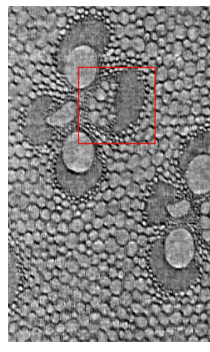

(b)

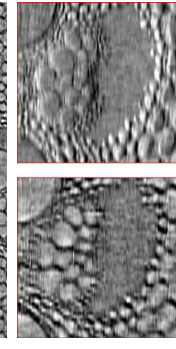

(c)

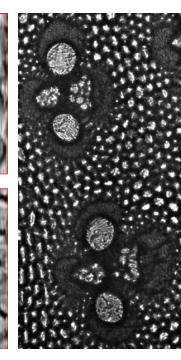

(d)
Fig. 5. Image of the bamboo sample: (a) differential phase and (b) amplitude images, quantitatively separated by using the laboratory set-up (scale bar $100 \mu \mathrm{m}$ ). (c) zoom of the highlighted $120 \times 120 \mu \mathrm{m}$ regions of (a) and (b), top and bottom respectively. (d) free space propagation image acquired with a high-resolution detector and monochromatic synchrotron radiation. 
conditions (with monochromatic synchrotron radiation and high resolution detector) is also shown for comparison in Fig. 5(d). We note that the higher contrast of this image is expected due to the much lower X-ray energy $(9.7 \mathrm{keV})$. The detail shown in the picture is similar but not exactly the same as in the laboratory image, due to difficulty in aligning the sample to the same region of interest.

In summary, we described a hard X-ray, laboratorybased, phase-contrast microscope, obtained through the appropriate design of a high-magnification edgeillumination XPCi system. The experimental set-up was built using commercially available, off-the-shelf instrumentation, and it is currently producing high quality amplitude and differential phase-contrast images. Micrometric spatial resolution was experimentally measured, theoretically described and numerically simulated by using a star pattern test object, with good agreement between simulation and experiment. By acquiring two separate images at different configurations, amplitude and differential phase can be quantitatively retrieved. The quantitativeness of the method was tested against theory on a two-material sample of known shape and composition. The instrument uses broadband and hard radiation, merging high resolution with the high penetration power of X-rays in a laboratory set-up.

\section{Acknowledgments}

The authors are grateful to Nikon Corporation and XTek, part of Nikon Metrology for their support with the experimental set-up. This work was supported by the UK Engineering and Physical Sciences Research Council (Grants EP/I022562/1 and EP/I021884/1). ME and PCD are supported by Marie Curie Career Integration Grants within the Seventh Framework Programme of the European Union, PCIG12-GA-2012-334056 and PCIG12-GA-2012-333990.

\section{References}

[1] R. Fitzgerald, Physics Today 53, 23 (2000).

[2] U. Bonse and M. Hart, Appl. Phys. Lett. 6, 155 (1965).

[3] K. Goetz, E. Foerster, P. Zaumseil, M. P. Kalashnikov, I. A. Mikhailov, G. V. Sklizkov, and S. I. Fedotov, Kvantovaia Elektronika Moscow 6, 1037 (1979).

[4] E. Foerster, K. Goetz, and P. Zaumseil, Kristall und Technik 15, 937 (1980).

[5] V. N. Ingal and E. A. Beliaevskaya, J. Phys. D Appl. Phys. 28, 2314 (1995).

[6] A. Snigirev, I. Snigireva, V. Kohn, S. Kuznetsov, and I. Schelokov, Rev. Sci. Instrum. 66, 5486 (1995).

[7] T. J. Davis, D. Gao, T. E. Gureyev, A. W. Stevenson, and S. W. Wilkins, Nature 373, 595 (1995).

[8] S. W. Wilkins, T. E. Gureyev, D. Gao, A. Pogany, and A. W. Stevenson, Nature 384, 335 (1996).

[9] A. Momose, T. Takeda, Y. Itai, and K. Hirano, Nat. Med. 2, 473 (1996)

[10] A. Olivo, F. Arfelli, G. Cantatore, R. Longo, R. H. Menk, S. Pani, M. Prest, P. Poropat, L. Rigon, G. Tromba, E. Vallazza, and E. Castelli, Med. Phys. 28, 1610 (2001).
[11] C. David, B. Nohammer, H. H. Solak, and E. Ziegler, Appl. Phys. Lett. 81, 3287 (2002).

[12] T. Weitkamp, A. Diaz, C. David, F. Pfeiffer, M. Stampanoni, P. Cloetens, and E. Ziegler, Opt. Express 13, 6296 (2005).

[13] F. Pfeiffer, T. Weitkamp, O. Bunk, and C. David, Nat. Phys. 2, 258 (2006).

[14] Z.-F. Huang, K.-J. Kang, L. Zhang, Z.-Q. Chen, F. Ding, Z.-T. Wang, and Q.-G. Fang, Phys. Rev. A 79, 013815 (2009).

[15] P. R. Munro, K. Ignatyev, R. D. Speller, and A. Olivo, Proc. Natl. Acad. Sci. U.S.A. 109, 13922 (2012).

[16] S. Mayo, T. Davis, T. Gureyev, P. Miller, D. Paganin, A. Pogany, A. Stevenson, and S. Wilkins, Opt. Express 11, 2289 (2003).

[17] M. Engelhardt, J. Baumann, M. Schuster, C. Kottler, F. Pfeiffer, O. Bunk, and C. David, Appl. Phys. Lett. 90, 224101 (2007).

[18] Y. Takeda, W. Yashiro, T. Hattori, A. Takeuchi, Y. Suzuki, and A. Momose, Appl. Phys. Express. 1, 117002 (2008).

[19] W. Yashiro, Y. Takeda, A. Takeuchi, Y. Suzuki, and A. Momose, Phys. Rev. Lett. 103, 180801 (2009).

[20] C. Holzner, M. Feser, S. Vogt, B. Hornberger, S. B. Baines, and C. Jacobsen, Nat. Phys. 6, 883 (2010).

[21] H. Kuwabara, W. Yashiro, S. Harasse, H. Mizutani, and A. Momose, Appl. Phys. Express. 4, 062502 (2011).

[22] J. Choi and Y.-S. Park, Appl. Phys. Express. 5, 042503 (2012).

[23] A. Olivo and R. Speller, Appl. Phys. Lett. 91, 074106 (2007).

[24] P. R. Munro, L. Rigon, K. Ignatyev, F. C. Lopez, D. Dreossi, R. D. Speller, and A. Olivo, Opt. Express 21, 647 (2013).

[25] P. R. Munro, C. K. Hagen, M. B. Szafraniec, and A. Olivo, Opt. Express 21, 11187 (2013).

[26] M. Marenzana, C. K. Hagen, P. D. N. Borges, M. Endrizzi, M. B. Szafraniec, K. Ignatyev, and A. Olivo, Phys. Med. Biol. 57, 8173 (2012).

[27] K. Ignatyev, P. R. T. Munro, R. D. Speller, and A. Olivo, Rev. Sci. Instrum. 82, 073702 (2011).

[28] T. Thuering, P. Modregger, T. Grund, J. Kenntner, C. David, and M. Stampanoni, Appl. Phys. Lett. 99, 041111 (2011).

[29] J. P. Guigay, C. R. Acad. Sc. Paris 284B, 193-196 (1977).

[30] J. P. Guigay, Optik 49, 121-125 (1977).

[31] F. A. Vittoria, P. C. Diemoz, M. Endrizzi, L. Rigon, F. C. Lopez, D. Dreossi, P. R. T. Munro, and A. Olivo, Appl. Opt. 52, 6940 (2013).

[32] M. Endrizzi, P. Oliva, B. Golosio, and P. Delogu, Nucl. Instr. Meth. Phys. Res. A 703, 26 (2013).

[33] P. R. T. Munro and A. Olivo, Phys. Rev. A 87, 053838 (2013).

[34] T. Schoonjans, A. Brunetti, B. Golosio, M. S. del Rio, V. A. Solé, C. Ferrero, and L. Vincze, Spectrochim Acta B 66, 776 (2011). 


\section{References}

[1] R. Fitzgerald, "Phase-sensitive x-ray imaging," Physics Today 53, 23-26 (2000).

[2] U. Bonse and M. Hart, "An x-ray interferometer," Appl. Phys. Lett. 6, $155-156$ (1965).

[3] K. Goetz, E. Foerster, P. Zaumseil, M. P. Kalashnikov, I. A. Mikhailov, G. V. Sklizkov, and S. I. Fedotov, "Measurements of the parameters of shell targets for laser thermonuclear fusion using an X-ray schlieren method," Kvantovaia Elektronika Moscow 6, 1037-1042 (1979).

[4] E. Foerster, K. Goetz, and P. Zaumseil, "Double crystal diffractometry for the characterization of targets for laser fusion experiments," Kristall und Technik 15, 937945 (1980).

[5] V. N. Ingal and E. A. Beliaevskaya, "X-ray plane-wave topography observation of the phase contrast from a non-crystalline object," J. Phys. D Appl. Phys. 28, 2314-2317 (1995).

[6] A. Snigirev, I. Snigireva, V. Kohn, S. Kuznetsov, and I. Schelokov, "On the possibilities of x-ray phase contrast microimaging by coherent high-energy synchrotron radiation," Rev. Sci. Instrum. 66, 5486-5492 (1995).

[7] T. J. Davis, D. Gao, T. E. Gureyev, A. W. Stevenson, and S. W. Wilkins, "Phase-contrast imaging of weakly absorbing materials using hard X-rays," Nature 373, 595-598 (1995).

[8] S. W. Wilkins, T. E. Gureyev, D. Gao, A. Pogany, and A. W. Stevenson, "Phase-contrast imaging using polychromatic hard x-rays," Nature 384, 335-338 (1996).

[9] A. Momose, T. Takeda, Y. Itai, and K. Hirano, "Phasecontrast x-ray computed tomography for observing biological soft tissues," Nat. Med. 2, 473-475 (1996).

[10] A. Olivo, F. Arfelli, G. Cantatore, R. Longo, R. H. Menk, S. Pani, M. Prest, P. Poropat, L. Rigon, G. Tromba, E. Vallazza, and E. Castelli, "An innovative digital imaging set-up allowing a low-dose approach to phase contrast applications in the medical field," Med. Phys. 28, 1610-1619 (2001).

[11] C. David, B. Nohammer, H. H. Solak, and E. Ziegler, "Differential x-ray phase contrast imaging using a shearing interferometer," Appl. Phys. Lett. 81, 3287-3289 (2002).

[12] T. Weitkamp, A. Diaz, C. David, F. Pfeiffer, M. Stampanoni, P. Cloetens, and E. Ziegler, "X-ray phase imaging with a grating interferometer," Opt. Express 13, 6296-6304 (2005).

[13] F. Pfeiffer, T. Weitkamp, O. Bunk, and C. David, "Phase retrieval and differential phase-contrast imaging with low-brilliance X-ray sources," Nat. Phys. 2, 258261 (2006).

[14] Z.-F. Huang, K.-J. Kang, L. Zhang, Z.-Q. Chen, F. Ding, Z.-T. Wang, and Q.-G. Fang, "Alternative method for differential phase-contrast imaging with weakly coherent hard x rays," Phys. Rev. A 79, 013815 (2009).

[15] P. R. Munro, K. Ignatyev, R. D. Speller, and A. Olivo, "Phase and absorption retrieval using incoherent X-ray sources," Proc. Natl. Acad. Sci. U.S.A. 109, 1392213927 (2012).

[16] S. Mayo, T. Davis, T. Gureyev, P. Miller, D. Paganin, A. Pogany, A. Stevenson, and S. Wilkins, "X-ray phasecontrast microscopy and microtomography," Opt. Express 11, 2289-2302 (2003).
[17] M. Engelhardt, J. Baumann, M. Schuster, C. Kottler, F. Pfeiffer, O. Bunk, and C. David, "High-resolution differential phase contrast imaging using a magnifying projection geometry with a microfocus x-ray source," Appl. Phys. Lett. 90, 224101 (2007).

[18] Y. Takeda, W. Yashiro, T. Hattori, A. Takeuchi, Y. Suzuki, and A. Momose, "Differential phase x-ray imaging microscopy with x-ray talbot interferometer," Appl. Phys. Express. 1, 117002 (2008).

[19] W. Yashiro, Y. Takeda, A. Takeuchi, Y. Suzuki, and A. Momose, "Hard-x-ray phase-difference microscopy using a fresnel zone plate and a transmission grating," Phys. Rev. Lett. 103, 180801 (2009).

[20] C. Holzner, M. Feser, S. Vogt, B. Hornberger, S. B. Baines, and C. Jacobsen, "Zernike phase contrast in scanning microscopy with x-rays," Nat. Phys. 6, 883887 (2010)

[21] H. Kuwabara, W. Yashiro, S. Harasse, H. Mizutani, and A. Momose, "Hard-x-ray phase-difference microscopy with a low-brilliance laboratory x-ray source," Appl. Phys. Express. 4, 062502 (2011).

[22] J. Choi and Y.-S. Park, "X-ray Phase Imaging Microscopy with Two-Dimensional Knife-Edge Filters," Appl. Phys. Express. 5, 042503 (2012).

[23] A. Olivo and R. Speller, "A coded-aperture technique allowing x-ray phase contrast imaging with conventional sources," Appl. Phys. Lett. 91, 074106 (2007).

[24] P. R. Munro, L. Rigon, K. Ignatyev, F. C. Lopez, D. Dreossi, R. D. Speller, and A. Olivo, "A quantitative, non-interferometric x-ray phase contrast imaging technique," Opt. Express 21, 647-661 (2013).

[25] P. R. Munro, C. K. Hagen, M. B. Szafraniec, and A. Olivo, "A simplified approach to quantitative coded aperture x-ray phase imaging," Opt. Express 21, 1118711201 (2013).

[26] M. Marenzana, C. K. Hagen, P. D. N. Borges, M. Endrizzi, M. B. Szafraniec, K. Ignatyev, and A. Olivo, "Visualization of small lesions in rat cartilage by means of laboratory-based x-ray phase contrast imaging," Phys. Med. Biol. 57, 8173 (2012).

[27] K. Ignatyev, P. R. T. Munro, R. D. Speller, and A. Olivo, "Effects of signal diffusion on X-ray phase contrast images," Rev. Sci. Instrum. 82, 073702 (2011).

[28] T. Thuering, P. Modregger, T. Grund, J. Kenntner, C. David, and M. Stampanoni, "High resolution, large field of view x-ray differential phase contrast imaging on a compact setup," Appl. Phys. Lett. 99, 041111 (2011).

[29] J. P. Guigay, "Analyse spectrale (frequences spatiales) d'une image de diffractionde fresnel," C. R. Acad. Sc. Paris 284B, 193-196 (1977).

[30] J. P. Guigay, "Fourier transform analysis of fresnel diffraction patterns and in-line holograms," Optik 49, 121-125 (1977).

[31] F. A. Vittoria, P. C. Diemoz, M. Endrizzi, L. Rigon, F. C. Lopez, D. Dreossi, P. R. T. Munro, and A. Olivo, "Strategies for efficient and fast wave optics simulation of coded-aperture and other x-ray phase-contrast imaging methods," Appl. Opt. 52, 6940-6947 (2013).

[32] M. Endrizzi, P. Oliva, B. Golosio, and P. Delogu, "Cmos aps detector characterization for quantitative $\mathrm{x}$ ray imaging," Nucl. Instr. Meth. Phys. Res. A 703, 26 $-32(2013)$.

[33] P. R. T. Munro and A. Olivo, "X-ray phase-contrast imaging with polychromatic sources and the concept of 
effective energy," Phys. Rev. A 87, 053838 (2013).

[34] T. Schoonjans, A. Brunetti, B. Golosio, M. S. del Rio, V. A. Solé, C. Ferrero, and L. Vincze, "The xraylib library for x-ray-matter interactions. recent developments," Spectrochim Acta B 66, 776 - 784 (2011). 